\title{
XXII. Über Composite. Composit-Zwillinge und -Viellinge.
}

\author{
Von \\ v. Goldschmidt in Heidelberg. \\ (Mit 3 Textfiguren.)
}

Es hat sich als wünschenswert gezeigt, eine Gruppe wichtiger, zum Teil bereits bekannter Gebilde unter einem gemeinsamen Namen zusammenzufassen. Es handelt sich um zusammengesetzte Krystalle, die ein geschlossenes Individuum bilden und unter Umständen, wie Einzelkrýstalle, zu Zwillingen und Viellingen zusammentreten.

Die Benennung: „zusammengesetzter Krystall wäre gut und bezeichnend für die in Rede stehenden Gebilde, doch ist sie zu compliciert. und besonders da unbequem, wo solche zusammengesetzte Krystalle zu Zwillingen, Drillingen, Viellingen zusammentreten, ebenso, als ob es einfache Krystalle wären.

Das statt dessen eingeführte Wort Composit ist die Übersetzung. Es wurde gewählt wegen seiner internationalen Verwendbarkeit und wegen seiner Fähigkeit, für die Zusammensetzung zu höherer Ordnung passende Wörter zu bilden. So kann man einen $Z$ willing aus zwei zusammengesetzten Krystallen einen Composit-Zwilling nennen, einen Drilling aus drei zusammengesetzten Krystallen einen Composit-Drilling, man kann von Composit-Viellingen, -Aggregaten, -Verwachsungen reden.

Das Wort erinnert an die analogen Gebilde im Pflanzenreich, die Compositen-Blüten. Auch im Tierreich gibt es analoge Gebilde, z. B. Korallen.

Beispiele. Quarz-Composite. Fast jeder Quarzkrystall, mag er äußerlich noch so einfach erscheinen, und noch so regelmäßige Messungen und genaue analytische Reinheit ergeben, zeigt sich bei der İtzung und Lösung als zusammengesetzt aus vielen, wir künnen sagen beliebig vielen 
Teilen, die gegen einander in Zwillingsstellung sind. Eines gegen das andere um die Hauptaxe um $60^{\circ}$ gedreht.

Die gleich gerichteten Teile bilden wohl zusammenhängende Gruppen,

Fig. 1.

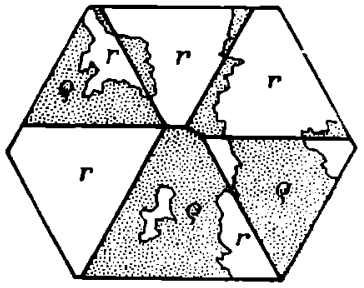

Quarz-Composit. füllen zusammenhängende Räume; doch haben diese Räume ganz unregelmäßige Abgrenzung. Auch steht nichts der Annahme im Weg, daB innerhalb dieser Räume ein Teil der Partikel, einzeln oder in kleineren Gruppen in Zwillingsstellung verdreht ist.

Unter den Quarzpartikeln, die den scheinbar einfachen Krystall, sagen wir den Composit, aufbauen, können Partikel von Rechtsund Linksquarz sein, diese unter sich parallel gerichtet, oder um $60^{\circ}$ in der Hauptaxe gedreht; man sagt in Zwillingsstellung $1 \%$. Wir begegnen da in Vereinigung und Abgrenzung einer unendlichen Mannigfaltigkeit.

Und doch erscheint uns solch ein Quarz-Composit als einheitlicher Krystall, ja als das Urbild des Krystalls, als der Bergkrystall, von dem unsere ganze Wissenschaft ausgegangen ist und den Namen erhalten hat.

Solche Quarz-Composite oder Composit-Quarze treten zu $\mathrm{Z}$ willingen mit geneigten Axen zusammen, ebenso, als ob es einfache Krystalle wären. Wir haben da mehrere Gesetze ${ }^{2}$ ) (Japaner, Reichenstein-Griesernthaler, Sardisches Gesetz).

Jeder solche Zwilling zeigt alle die Eigentümlichkeiten einfacher Zwillinge (Abplattung, Induction, Beeinflussung an der $Z$ willingsgrenze u. a.) und doch ergibt die strengere Untersuchung, daß jedes der beiden Individuen ein sehr complicierter Composit ist.

Hier zeigt sich nun der Vorteil, ja die Notwendigkeit, der Einführung eines Begrifls (für den das Wort Composit vielleicht nicht unpassend ist), der uns ermöglicht von $Z$ willingen zu reden, indem wir jedes der beiden vereinigten Gebilde als Ganzes, als Individuum auffassen, anderen gegenüber, andererseits als einen viellingsmäßig gegliederten Bau in sich.

Man könnte sagen: Composit ist ein Individuum nach außen, ein Vielling (oder sonst ein gegliederters Ganze) in sich.

Wir sprechen von einem Quarzkrystall und lassen es dabei unentschieden, ob dieser sich bei strengerer Prüfung als ein Composit herausstellt.

1) Vergl. diese Zeitschr. 1898, 30, 257.

2) Vergl. Min.-petr. Mitt. $1905,24,167$. 
Analog sprechen wir von einer Blüte, unbekümmert, ob diese sich bei genaucrer Betrachtung als eine zusammengeselzte, eine composite herausstellt. Auch bei den Früchten ist es so. Díe Heidelbeere ist eine Einzelbeere, die Hímbeere ein Composit und doch nennen wir jede eine Frucht.

Auch in der Zoologie finden wir das Analoge. So bildet das Insektenauge eine Mosaik von Einzelaugen, man kann es als einen Compositen ansehen und doch möchte man nicht darauf verzichten, es als (janzes ein $\Lambda$ uge zu nennen und zu sagen, die Fliegen haben ein Paar Augen, ebenso wie wir oder wie die Frösche.

Jer Fall Quarz steht nicht vereinzelt da. Es gibt viele solche Fälle. Ja man kann nie sicher sein, daß nicht irgend ein Krystall von größter Reinheit und Homogenität von scheinbar einfachstem Bau sich bei feinerer Untersuchung als ein Composit herausstellt. Es könnte soweit kommen, daß man zweifelhaft wird, ob es einfache Krystalle in aller Strenge gibt, ob nicht alle sich als Composite zeigen würden, wenn man nur fein genug untersuchen künnte. Andererseits würde man eine Verwirrung in die Krystallographie bringen, wollte man alle die nachgewiesenen oder möglichen zusammengesetzten Krystalle von den Individuen ausscheiden.

Als Auswcg erscheint eben die Einführung unseres Begriffs. Composit ist ein Individuum, ein Einzelkrystall, aber ein in sich gegliederter. So kann man die Begriffe Krystall, Einzelkrystall, Individuum festhalten und anwenden mit dem geistigen Vorbehalt, daß das Individuum sich als ein Composit herausstellen könnte.

\section{Weitere Beispiele.}

Feldspat-Composite. Es gibt vielleicht auf der Welt keinen Albitkrystall, der einheitlich wäre. Jeder zeigt makroskopisch oder mikroskopisch einen Aufbau aus Zwillingslamellen, vereinigt nach dem Albitgesetz. Die Lamellierung geht ins Feinste hinab und wir können annehmen, daß sie sich fortsetzt, wo das Mikroskop versagt.

Dabei bilden die einzelnen Albitindividuen (die sämtlich Composite nach dem Albitgesetz sind) Zwillinge nach dem Periklingesetz, dem Karlsbader, dem Bavenoer Gesetz. Sic sitzen regelmäßig geordnet auf dem Orthoklas oder Mikroklin, der selbst ein Composit ist.

Wollten wir auf den Begriff des zusammengesetzten Krystalls, des Composit-Individuums, verzichten, so gäbe es überhaupt keine Albitkrystalle, keine Albitzwillinge, sondern nur sehr complicierte, ja unauflüsbare Viellinge.

Beim Mikroklin zeigt das Polarisationsmikroskop das Krystallindividuum, bestehend aus einem äußerst feinen System von Lamellen, die sich durchkreuzen. Es ist dabei unentschieden, ob die Lamellen unter sich gleich und homogen sind, oder ob Orthoklas und Albit particulär oder in Schichten alternieren und sich mischen. Manchmal sind Lamellen und 
Bänder von Albit im Mikroklin ausgeschieden, unter dem Polarisationsmikroskop als Composit-Viellinge nach dem Albitgesetz erkennbar.

Und doch können wir nicht darauf verzichten, den Mikroklin trotz seines complicierten Viellingsbaues als Krystallindividuum aufzufassen. Diese

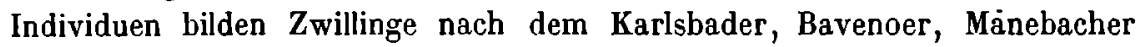
Gesetz, gerade so wie die einfachen Adulare.

Man kann nicht wissen, ob die scheinbar einfachen Adulare sich nicht eines Tages bei verfeinerter Untersuchung ebenfalls als Composite herausstellen. Wäre das der Fall, so gäbe es überhaupt keinen einfachen Feldspat mehr; denn die Plagioklase dürften alle zusammengesetzt sein. Dafür spricht die petrographische Untersuchung, die alle Plagioklase an der Viellingsstreifung erkennen läßt.

Man findet oft Albite orientiert auf Orthoklas und Mikroklin sitzen. Manchmal überziehen sie dieselben rundum wie mit einer Haut. Die Mikrokline sind Composite, ebenso wie die Albite. Es fragt sich (begrifflich), ob man das Ganze als einen Compositen auffassen soll, oder als die regelmäßige Verwachsung zweier Composite. Dabei ist zu untersuchen, ob nicht jedesmal, wo Albit sich orientiert aufsetzt, dem Orthoklas oder Mikroklin Albitteilchen orientiert eingeordnet sind, dem sich der neu zutretende Albit orientiert anlegt.

Zinkblende-Composite. Scheinbar einfache Krystalle von Blende sind von Viellingslamellen nach dem Blendegesetz durchsetzt. (Drehungsaxe $\perp p=1)$. Fast jeder Blendekrystall zeigt solche, ebenso jedes Spaltstück, selbst die reinsten von Santander. Die Lamellen erscheinen einzeln oder in Scharen bis zu mikroskopischer Feinheit. Nach einer der vier Tetraëderflächen oder nach allen, so daB die Systeme sich kreuzen $\left.{ }^{1}\right)$. Diese Systeme lassen sich durch Ätzung wahrnehmen; an Spaltstücken, schöner noch an Kugeln.

Die Blende-Composite treten als Ganzes zu Zwillingen, Drillingen, Viellingen nach dem Blendegesetz zusammen, wie einfache Krystalle, von denen sie sich äußerlich nicht unterscheiden.

Eisen-Composite. Die Meteoreisen bilden Einzelkrystalle, durchsetzt von Lamellen in großer Zahl. Diese Lamellen erscheinen auf geätzten Platten als die Widmannstättenschen Figuren. Sie verlaufen parallel den Oktaëderflächen, manchmal auch nach anderen Krystallflächen. Sie sind grob bis mikroskopisch fein. Zwischen den Eisenblättern sitzen Lamellen von etwas anderer Zusammensetzung.

Nach der Deutung von Linck ${ }^{2}$ ) sind die Lamellen des oktaëdrischen Eisens Viellingslamellen nach dem Spinellgesetz nach den vier Oktaëder-

1) Vergl. Ph. Hochs child, Dissert. 1907.

2) Linck, Ann. Wien. Mus. 1893, 8, 113. 
flächen, wobei die Zwillingsebene zugleich Verwachsungsebene ist. Es sind danach die Meteoreisen als Viellings-Composite anzusehen.

Auch bei den Meteoreisen wurde gezeigt, daß größere in sich lamellierte Partien zu einander als Ganzes in Zwillingsstellung stehen. Wir kennen also auch hier Composit-Zwillinge ${ }^{1}$ ).

Caloit-Composite. Man kennt von Andreasberg Durchkreuzungszwillinge und -viellinge von Calcit von rhomboëdrischem Habitus von bedeutender Größe, die an anderem Ort eingehender beschrieben werden sollen 2). Jedes der sich durchkreuzenden Individuen bildet einen ViellingsComposit, aufgebaut aus unzähligen papierdünnen bis mikroskopischen Blättchen, die in sich und unter sich nach dem Basisgesetz verzwillingt sind. Hand in Hand mit dieser Viellingsbildung geht eine Absonderung nach der Basis, die von einem prächtigen Perlmutterglanz oder Seidenglanz begleitet ist, wie er sonst dem Calcit nicht eigentümlich ist.

Der Perlmutter- oder Seidenglanz und die Absonderung (Pseudospaltbarkeit) nach der Basis charakterisieren diese schönen und eigenartigen Composite.

Eine zweite Erscheinungsform solcher Kalkspat-Composite sind Tafeln, ausgeplattet nach der Basis, teils drei- oder sechsseilig, teils unregelmäßig begrenzt, bedeckt mit Dreiecken in Zwillingsstellung. Auch sie zejgen den schünen und charakteristischen Seidenglanz und die Absonderung nach der Basis.

Die gleiche Oberflächenzeichnung wie die Platten verbunden mit demselben mondsteinähnlichen Perlmutterglanz, zeigen Krystalle von Andreasberg, bei denen Prisma und Basis herrschen. Sie dürften in gleicher Weise als Viellings-Composite nach der Basis zu deuten sein. Sie bilden Zwillinge mit geneigten Axen. Dieselben sollen an anderem Ort eingehender beschrieben werden.

Die gleiche Erscheinung zeigt sich, im einzelnen modificiert, bei Calciten von Guanajuato und Zacatecas. Auch wurden solche Calcite von Hessenberg ${ }^{3}$ ) und vom Rath vom Maderaner und Ahrn-Tal beschrieben.

Die Calcit-Composite von Andreasberg und anderen Orten bilden $Z$ willinge mit paralleler, ebenso mit geneigter Hauptaxe, die alle Eigentümlichkeiten einfacher $\mathrm{Z}$ willinge zeigen.

Calcit. Viellings-Composite nach $-\frac{1}{2}=-\frac{1}{2} \mathbf{R}$. Viele Calcit-

1) Berwerth, Wien. Sitz.-Ber. 1902, 111 (1), 646. Diese Zeitschr. 1905, 40, 619.

2) Vergl. Hessenberg, Senckenb. Abh. 1875, 10, Sep., S. 21, Taf. 2, Fig. 11.

3) Hessenberg, Senckenb. Abh. 1862, 4, 9 u. 13.

vom Rath, Pogg. Ann. 4875, 155, 48. 
krystalle zeigen einzelne Lamellen in Zwillingsstellung nach dem Rhornboëder - $\frac{1}{2}$. Einzeln oder in parallelen Scharen; nach einer Richtung oder sich kreuzend nach zwei oder drei Flächen von $-\frac{1}{2}$. Man bemerkt sie, wenn einzeln, im wasserhellen Krystall durch das Farbenspiel beim Durchschauen in passender Richtung, wenn in Schaaren auftretend, an einer Streifung der Oberfläche, an der schönen Lichterscheinung, die Kobelli) beschrieben hat.

Wie weit diese $Z$ willingslamellen verbreitet sind, können wir daran erkennen, daß es dem Optiker schwer wird, aus den reinsten Partien des reinsten uns bekannten Calcit, des Isländer Doppelspat, Stücke herauszuschneiden, die nicht durch diese Lamellen für seine Zwecke verdorben sind.

Auch diese Composite bilden, wie Einzelkrystalle, Zwillinge, Drillinge, nach allen Gesetzen, die dem Calcit eigen sind. Wir sind genütigt sie trotz ihrer polysynthetischen Einlagerungen als Einzelkrystalle zu behandeln. Wollten wir das nicht, so kïnnte es geschehen, daß es bei strenger Prüfung vom Calcit überhaupt keine Einzelkrystalle gäbe.

Glimmer- und Chlorit-Composite. Die ausgezeichnete Spaltbarkeit und die Absonderung nach der Basis, dazu der eigenartige Glanz (der dem Glimmer den Namen verschafft hat) gehen Hand in Hand mit einer Viellingsbildung nach der Basis. Die Partikel gegen einander um $60^{\circ}\left(180^{\circ}\right)$ gedreht, in Schichten oder innerhalb der Schicht in Gruppen alternierend. Damit im Zusammenhang das optische Verhalten.

Mit der Compositbildung nach einer Vorzugsfläche (z. B. der Basis) ist, außer der Absonderung, häufig eine tafelige Ausbreitung nach dieser Fläche verbunden. So beim Calcit und Eisenglanz. Es bleibt zu untersuchen, ob nicht die Eigenart der glimmerartigen Mineralien (Glimmer, Chlorit, Graphit, Uranglimmer, Eisenglimmer u. a.) gerade durch die Compositbildung bedingt ist.

Fig. 2.

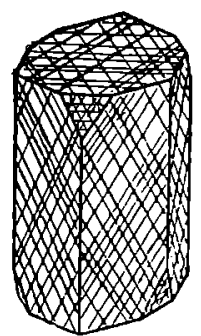

Fig. 3.

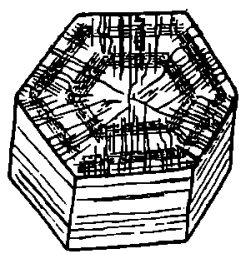

Korund - Composite. Wir lesen bei Bauer2):

¿Zwillinge gibt es: 1. nach $R$ : Meist sind einzelne Lamellen nach den Rhomboëderflachen einem größeren Krystall in Zwillingsstellung nach $R$ eingewachsen; dadurch entsteht häufig eine schalige Absonderung in der Richtung der Flichen von $R$, welche man nicht mit Blätterbrüichen verwechseln darf; sie ist entweder nur einem oder zwei resp. allen drei Flächen $R$ parallel; dann vielfach ausgezeichnete Viellingss/reifung über die ganze Krystallfäche hin.e

28. Nach der Basis $c$ : eine Anzahl düner Lamellen liegt nach dieser Flache

1) K obell, Erdm. Journ. 1862, 86, 466, sowie Taf. 2, Fig. 9.

2) Bauer, Lehrb. d. Min. 190\%, 510. 
zwillingsartig über einander, die abwechselnden in paralleler, die anstoßenden in Zwillingsstellung und rufen auch in dieser Richtung zuweilen schalige Absonderung und auf den Prismenflächen eine horizontale Streifung hervor.e

Das ist das charakteristische Bild von Compositen. Die S. 352 beigefügten aus Tschermaks Jehrb. d. Min. entnommenen Figuren mögen es illustrieren. Auch das abnorme optische Verhalten ist eine charakteristische Folge der Compositbildung.

Wir lesen diesbezüglich bei Melczer ${ }^{1}$ ):

\L asa ulx hal gefunden, daß die optische Zweiaxigkeit dieses Minerals mit dem Vorhandensein der Zwillingslamellen nach den Flächen des Grundrhomboëders und mit der schaligen Zusammenselzung nach den Prismenflä(hen im Zusammenhang steht, indem solche Krystalle, an denen Obiges nicht vorhanden ist, ein ungestörtes einaxiges Bild liefern. Ich kann die Beobachtungen von Lasaulx durch meine Erfahrungen an Birmaner Krystallen nur bestătigen. .

Auch die Erscheinungen am Sternsaphir dürften als Folgen der Composition anzusehen sein.

Ferner beobachten wir am Korund wohl als Folge der Compositbildung nach der Basis (analog dem Quarz) das gleichzeitige $\Lambda$ uftreten desselben Rhomboëders oder Skalenoëders im + und - Sextanten zugleich, z. B.: $\pm 1, \pm \frac{1}{2} ; \pm 41$ u. a. Dadurch wird die Unterscheidung der \pmFormen erschwert, oft unmöglich gemacht. Der gleichen Schwierigkeit begegnen wir beim Quarz ${ }^{2}$. Auch dort ist die Compositbildung die Ursache.

Harmotom und Phillipsit bilden Drillinge und Vicllinge aus zusammengesetzten Individuen (Composit-Drillinge). Die Art des Aufbaues ist Gegenstand mehrfacher Studien gewesen.

Mimetische Krystalle sind nach Tschermak ${ }^{3}$ ), der diesen Begriff aufgestellt hat, »aus vielen (pseudosymmetrischen) Individuen in complicierter Weise aufgebaute Zwillingsbildungen, welche die Formen eines Krystallsystems höherer Ordnung nachahmen «. Dabei sind:

Pseudosymmetrische Krystalle $\left.{ }^{3}\right)$. Einzelkrystalle, $>$ die durch gleichzeitige Ausbildung ungleichartiger Flächen Combinationen zeigen, welche einer höheren Symmetrie zu entsprechen scheinen ".

Der Begriff der mimetischen Krystalle deckt sich nicht mit unserem Begriff der Composite. Letzterer ist der weitere Begrifi. Er erfordert nicht, daß die \%usammenselzung Gebilde von scheinbar höherer Symmetrie hervorbringt. Sonst wären Composile aus oktaëdrischen Iamellen nicht denkbar, da ja das Oktaïder bereits die hüchste Symmetric besitzt. Auch sind nicht nur die pseudosymmelrischen Krystalle zur Compositbildung

1) Melczer, diese Zeitschr. 1902, 35, 562.

2) Vergl. Gdt., Intex 1891, 3, 4.

3) Tschermak, Lehrl). d. Min. 1897, 91. 
geeignet und geneigt, sondern auch die höchst symmetrischen, die Krystalle des regulären Systems, auch die holoëdrischen. Es ist ferner bei den Compositen die Form des zusammengesetzten Gebildes nicht erst durch die regelmäßige Gruppierung der verschieden gerichteten Subindividuen hervorgebracht. Es kann vielmehr ein Einzelindividuum dem Compositen die Gestalt geben und die anders orientierten Teile als Lamellen oder Enclaven einschließen. Dies ist ein hüufiger Fall, wenn nicht der häufigste.

Ein häufiger Fall der Composition ist der folgende: Die Teile in Zwillingsstellung bringen gemeinsam eine Gestalt hervor, die jeder in beiden Stellungen des Zwillings annehmen kann. Also eine Form, die aus Deckflächen des Zwillings besteht. Wir könnten sagen eine Deckform.

Beispiele. Die meisten Quarz-Composite zeigen Prisma und Pyramide, strenger das Prisma $\infty 0$ und beide Rhomboëder \pm 10 . Diesc, zusammen auftrelend, decken sich bei den Zwillingen mit gemeinsamer Hauptaxe. Bei den Calcit- und Glimmer-Compositen nach der Basis ist die luäuligste Form die Basis mit hexagonaler Begrenzung, die den Individuen beider Stellung gemeinsam ist.

Bestimmend für die regelmäßige Verwachsung, somit auch für die Compositbildung sind die Hauptrichtungen (Hauptflächen, Hauptknoten, Hauptzonen, Hauptattractionsrichtungen). Die Nebenrichtungen (Nebenflächen, ...) werden mitgeführt $\left.{ }^{1}\right)$. So verwachsen die Quarzpartikel nach den IIauptknoten $\infty 0$ und \pm 10 ohne Rücksicht auf die schwachen Knoten, die abgeleiteten Rhomboëder, rechten und linken Trapezoëder.

Compositbildung ist daher besonders häufig bei schwacher Hemiëdrie, d. h. da, wo die Ilauptknoten im Zwilling gleich gerichtet und nach Art und Stärke der Betätigung nur wenig verschieden sind. Bei solchen wird die Ilemiëdrie nur unter besonders günstigen Umständen erkannt, der Composit für ein einfaches Individuum gehalten.

Heteroaxiale Composite. Als heteroaxiale Verwachsung bezeichneten wir eine Verknüpfung der Partikel durch Parallelrichten ungleicher Kräfte (diese Zeitschr. 1898, 29, 377). Dort war auch von heteroaxialen Mischkrystallen die Rede. Es wurde gesagt:

•Sind die Kräfle ähnlich (zugleich auch wohl die Winkel), so können sich die Anheftungsaxen von Partikel zu Partikel vertauschen. Das Resultat ist ein scheinbar einfacher, aber mit gewissen linregelmüßigkeiten behafteter Krystall. Wir wollen ihn theteroaxialen Mischkrystall a nennens (vergl. Index 1891, 3, 136).

Wir wollen jetzt diese Gebilde zu den Compositen rechnen und sagen: IIeteroaxiale Composite sind durch heteroaxiale Verwachsung gebildete Composite.

Grenzen des Begriffs. Es fragt sich, wie weit man den Begriff ausdehnen soll. Soll man zu ihm nur die Verwachsung gleichartiger

1) Vergl. Gdt., Mineralog.-petr. Mitt. $1905,24,168$. 
Partikel rechnen, soll man die enantiomorphen und isomorphen hereinbeziehen oder auch ungleichartige Partikel?

Die enantiomorphen darf man gewiß nicht ausscheiden, so die Verwachsung von Rechts- und Links-Quarzpartikeln. Aber auch die isomorphen muß man hereinbeziehen. Sonst hïtte man z. B. alle Feldspäte wegzulassen. Desgleichen dürften die regulïren Viellingsverwachsungen heterogener Partikel hereinzubezichen sein; so die Durchdringung von Eisenglanz mit Rutil, von Chlorit, Eisenglanz, Rutil mit Glimmer, von Kup, ferkies mit Blende u. a.

Ziehen wir diese herein, so erhallen wir folgende Classification der Composite:

1. Viellings-Composite. 3. Isomorphe Composite.

2. Heteroaxiale -

4. Heterogene -

Auszuschließen sind fremdartige, nicht orientierte Einschlüsse, z. B. Quarzsand in Calcit (Sandkrystalle), Quarze mit unregelmäBigen Einschlüssen von Rutil, Güthit, Chlorit u. a. Ferner sind auszuschließen: Pseudomorphosen und Paramorphosen.

Nach Art der Composition können wir unterscheiden:

1. Lamellare Composite (nach einer oder mehreren rlïchen).

2. Unregelmüßig in einander greifende (z. B. Quarz) und zwar verzahnte und Enclaven bildende.

3. Zonare Umhüllungen (besonders bei isomorphen).

4. Composite nach Wachstumskegeln.

5. Particular gemischte Composite.

Heidelberg, Januar 1907. 